\title{
Impacts of Parental Involvement and Parents' Level of Education on Student's Academic Accomplishment
}

\author{
Won-Tack Lim \\ Margaret Warner Graduate School of Education and Human Development, University of Rochester, Rochester, USA
}

\section{Email address:}

wlim4@u.rochester.edu

\section{To cite this article:}

Won-Tack Lim. Impacts of Parental Involvement and Parents' Level of Education on Student's Academic Accomplishment. Education Journal. Vol. 10, No. 1, 2021, pp. 35-39. doi: 10.11648/j.edu.20211001.15

Received: February 10, 2021; Accepted: February 20, 2021; Published: February 26, 2021

\begin{abstract}
Parental involvement in school has been demonstrated to play a crucial role in children's academic achievement. This article examines the relation of adolescent's academic achievement to parental involvement and parent's education level, separately, grounded on a quantitative approach. All participants were middle-class dual-earner parents from eight cities across the United States. Spearman's correlation coefficient was used to find out if adolescent academic performance is correlated with parental involvement, and Mann-Whitney U test was utilized when children's academic performance was compared between groups of parents with different educational levels. Results show that children's academic achievement is only correlated with certain dimensions of parental involvement. Results further show that, across the board, children's school achievements do not significantly differ across the groups with different levels of parents' education if parents in all groups are equally committed to children's education. As a result, this study concludes that the effect of parental involvement on children's academic success differs by the type of parental engagement and that parents' level of education does not carry much weight over children's academic performance when all parents are actively involved in their schooling. Both limitations and suggestions for future studies are also discussed.
\end{abstract}

Keywords: Parental Involvement, Academic Achievement, Education Level, Quantitative Approach, Middle-Class Dual-Earner Parents

\section{Introduction}

Parents play a key role in their children's education. In a general sense, parental involvement indicates a wide array of practices that are aimed at enhancing children's educational accomplishment and motivation [1]. Bloom [2] operationally defined parental involvement as parental aspirations for their children's academic achievement. Parental involvement has positive effects on students' academic achievement, attitude, and motivation [3-5]. A myriad of studies demonstrated that academic success improves when parents are engaged and invested in their children's schooling $[3,5]$. Increased parental involvement has also been clearly linked to more positive student behaviors and attitudes toward school. On the other hand, when parental involvement or resources are absent or not fully provided in children's education, it can often lead to low academic performance [6]. As such, it is fair to say that the level of parental involvement considerably affects how successful their children's academic lives can be.

In addition to the parental involvement, children's school achievement is to a great extent linked to parents' educational background itself as well. Parents' education level has been regarded as one of the strong predictors of their children's academic achievement [7-9]. Simply put, it indicates that the higher the academic degree earned by parents, the higher the school grade received by their children. Khan and colleagues [8] examined how much parents' educational level affects students' academic achievements of the secondary school level in Pakistan. The researchers randomly recruited 200 students in the 10th grade in District Rajanpur and utilized direct observation, interviews, and survey questionnaires to gather information about personal and family background, and used the results of the 9th grade class in secondary school certificate examination as an indicator that represents their level of academic performance. Their findings revealed that there is a significant positive impact of parents' education level on 
academic learning of secondary school students. Such a positive association can be accounted for by parents' certain beliefs and behaviors [10]. Parents with stronger educational backgrounds tend to have higher confidence in their children's academic performance as well as higher expectations of their children [10]. These high expectations motivate children to perform well, and the confidence the parents keep in their children creates their own confidence to excel in class, which may lead them to become more successful academically. In that regard, parents' level of education could indeed predict their child's success in his academic abilities, to a certain degree.

In the present paper, secondary data analysis from a family study conducted in 1998-2000 was utilized [11], examining two hypotheses concerning the correlation of a student's academic performance (K-12) with parental involvement as well as with parents' level of education completed. The dataset used in this previous study was particularly selected for the current investigation due to the distinctive characteristics of socioeconomic status (SES) of the conducted study population, who were the middle-class, dual-career families living in the United States. Previously, research has focused on exploring how parental involvement impacts children's academic performance primarily in low-income families, with the middle-class, dual-career families being excluded from the mainstream research focus. With the given data, hence, the primary focus of the current study was on identifying the interrelationship of parental involvement with their children's academic accomplishment under this particular population.

Prior to the current study, it was initially predicted that adolescents whose parents are highly involved in their academic and social lives would perform better on tests than those whose parents are not, as consistent with the results of previous research. Then, the focus shifted to probe how adolescent academic achievement would differ according to the education level of their parents when equal levels of parental involvement are provided. Although there has been a large volume of research that solely focuses on the relationship between student's academic achievement and parents' educational level, no published studies attempt to examine whether different levels of parents' education would lead to significant differences in children's school achievement under the condition that the same amounts of parental involvement are ensured. The initial prediction in this regard was that adolescents' grades would be higher when highly educated parents are engaged in their child's schooling compared to when lowly or medium educated parents are. Having reflected on such predictions, two hypotheses were established as follows:

Hypothesis I: There is a positive correlation between children's academic accomplishment and parental involvement.

Hypothesis II: Children perform better academically when parents with higher levels of education are involved in their education than when parents with lower levels of education are.
In this study, "higher levels of education" and "lower levels of education" refer to schooling beyond high school and schooling up to high school, respectively.

\section{Method}

\subsection{Participants}

All participants $(\mathrm{N}=878)$ were middle-class dual-career parents of children aging from 11 to 19, from eight cities across the United States. Five sites are located in the Midwest (Maple, Forest Bluff, Kingston, Mettawa, and Cedar), one site is located in the Southeast (Feldnor), one site is situated in the Northeast (Middle Brook), and one is situated on the West Coast (Del Mar). Apportioned by gender, 376 (42.8\%) men and $502(57.2 \%)$ women participated. All participants were dual-career, middle-class families. $84.4 \%$ of the respondents were predominantly White, followed by Black (6.7\%), Hispanic (2.8\%), and Asian or Pacific Islander (1.8\%).

\subsection{Measures}

The current investigation used secondary data that was collected from 1998 to 2000 to examine the complex dynamics of modern families [11]. In this family study, fathers, mothers, and children were requested to complete multiple instruments comprised of surveys, in-depth interviews, and time diaries. Such instruments were used to collect information regarding marriage, work, childrearing and parental supervision, management of domestic chores, time distribution, coping strategies, and mental wellness. The surveys were sent home to complete and returned by mail. For the purpose of the current inquiry, only parent survey questionnaires were utilized for analysis.

The questionnaire consists of open-ended questions, closed-ended questions, Likert scale questions, and demographic questions. There are 96 questions asking about family's background, perceptions of family life, household chores, relationship with spouse, teenager's life, personal well-being, and demographic information such as education, income, and work life. Of all the questions, seven questions were drawn and used for statistical analysis of the present research, one of which is concerned with parents' highest level of education, two with children's academic achievement, and four with parental involvement.

The question about the level of parental educational attainment is stated "What is the highest degree or level of school you and your spouse/partner have completed?" with responses on a 4-point Likert scale (1=less than high school, 2=high school level, 3=undergraduate level, 4=graduate/professional level). In this study, each response from parent and his/her spouse was incorporated, and then their median values were calculated and used as the highest level of education representing both parents. The two questions concerning children's school performance are stated "Which of the following best describes the grades your teen received on his/her last report card?" and "Since the beginning of the school year, has your teen received an award for his/her 
academic performance?". Responses were made with a 5-point Likert scale ( $1=$ mostly As, $2=$ about half As and half Bs, $3=$ mostly Bs, $4=$ about half Bs and half $\mathrm{Cs}, 5=$ mostly $\mathrm{Cs}$ or below) and with a dichotomous choice ( $1=$ Yes, $2=$ No), respectively.

In the original questionnaire, there are 13 questions relating to parental educational involvement, and Cronbach's $\alpha$ for these items is .74. However, excluding duplicate questions or questions that are barely related to the study focus, only four essential items were extracted and eventually used for this research, which are as follows: "How many hours per week do you personally spend on taking the kids to and from activities?" ( $1=0$ hours, $2=1-2$ hours, $3=3-5$ hours, $4=6$ or more hours), "How many hours per week do you personally spend on helping the kids with their homework?" (1=0 hours, $2=1-2$ hours, $3=3-5$ hours, $4=6$ or more hours), "How often do you and the teen in the study participate in talking about everyday events in your lives?" ( $1=$ seldom or never, $2=$ less than once a week, $3=$ once a week or more often), and "How often do you and/or your spouse /partner check on whether your teen has done his/her homework?" (1=seldom or never, $2=$ occasionally, $3=$ often). In previous studies, parental involvement was deemed as parents' participation in schooling activities [12], so the first two questions were designed to measure such engagement of parents. Christenson et al. [13] and Walberg [14] equated parental involvement with parents' communication with their children about education and schoolwork, and the third question is associated with such dimension of parental involvement. The fourth question is concerned with parents' supervision of children, which is another definition of parental involvement $[15,16]$.

\subsection{Statistical Analyses}

Spearman's rank correlation coefficient was calculated to examine the relationship between parental involvement and children's school performance. Mann-Whitney U test was employed to compare the children's academic performance of highly educated parents with that of low- and medium-educated parents in the case where the same amounts of parental involvement are provided. For this statistical procedure, only parents who devoted an equal amount of time/frequency to their child's education from both groups, higher education level and secondary education level, were selected for a comparative analysis (e.g., only respondents who answered "often" to the question asking about the frequency of parents checking on whether their children have finished his/her homework).

\section{Results}

\subsection{Results of the First Hypothesis Test}

The results of the Spearman's correlation analysis are presented in Table 1. It was found that teen's grade was positively correlated with time of parents spending on helping their homework $(\mathrm{r}=.13, \mathrm{p}<.05)$. A positive correlation was also found between teen's academic award and frequency of parents talking about everyday events with children $(\mathrm{r}=.10, \mathrm{p}$ $<.05)$. Frequency of parents checking their child's homework was significantly positively correlated with teen's grade ( $\mathrm{r}$ $=.31, \mathrm{p}<.01)$ and was significantly negatively correlated with teen's academic award $(\mathrm{r}=-.19, \mathrm{p}<.01)$. This indicates that the more often parents checking their child's homework, the better grade teen will likely get, but at the same time, the less likelihood teen will receive an academic award. No other parental involvement variables showed a significant correlation with the measures of children's academic accomplishment. As a result, the first hypothesis that children's academic achievement is positively correlated with parental involvement was only partially verified.

Table 1. Spearman's correlation coefficients for study measures.

\begin{tabular}{|c|c|c|c|c|c|c|}
\hline Variable & 1 & 2 & 3 & 4 & 5 & 6 \\
\hline 1. Time parents help kid's homework & - & & & & & \\
\hline 2. Time parents take kids to activities & $.34^{* *}$ & - & & & & \\
\hline 3. Frequency parents check kid's homework & $.23^{* *}$ & .08 & - & & & \\
\hline 4. Frequency parents talk everyday events & $.09^{* *}$ & $.09^{* *}$ & .08 & - & & \\
\hline 5. Teen grade & $.13^{*}$ & .02 & $.31^{* *}$ & -.09 & - & \\
\hline 6. Teen academic award & -.02 & .03 & $-.19^{* *}$ & $.10^{*}$ & $-.43^{* *}$ & - \\
\hline
\end{tabular}

${ }^{* *} p<.01,{ }^{*} p<.05$.

\subsection{Results of the Second Hypothesis Test}

Mann-Whitney U test was administered to compare teen school performance of parents who completed secondary education with that of parents who completed higher education when equal amounts of parental involvement are given, and the relevant results are summarized in Table 2. It was confirmed that grades of children whose parents completed secondary education were statistically significantly higher than those of children whose parents completed higher education when parents in both groups often engaged in talking about everyday events with their children $(\mathrm{U}=2030.50, \mathrm{p}<.01)$. No other significant difference was found between parents' educational level and teen school achievement in relation to different dimensions of parental involvement. These results did not support the second hypothesis that children do better academically when highly educated parents are involved in their education than when low- and medium-educated parents are involved. 
Table 2. Mann-Whitney U Test for Parents' Educational Level and Other Variables.

\begin{tabular}{|c|c|c|c|c|c|c|}
\hline Variables & Parents' educational level & $n$ & Mean rank & Sum of ranks & $\boldsymbol{U}$ & $Z$ score \\
\hline \multicolumn{7}{|l|}{ 1. Teen grade } \\
\hline \multirow{2}{*}{ Time parents help kid's homework (3hrs or more) } & Secondary education & 4 & 13.50 & 54.00 & \multirow[t]{2}{*}{44.00} & \multirow{2}{*}{-.27} \\
\hline & Higher education & 24 & 14.67 & 352.00 & & \\
\hline \multirow{2}{*}{ Time parents take kids to activities (3hrs or more) } & Secondary education & 6 & 15.00 & 90.00 & \multirow[t]{2}{*}{69.00} & \multirow[t]{2}{*}{-.94} \\
\hline & Higher education & 30 & 19.20 & 576.00 & & \\
\hline \multirow{2}{*}{ Frequency parents check kid’s homework (often) } & Secondary education & 17 & 65.00 & 1105.00 & \multirow[t]{2}{*}{595.00} & \multirow[t]{2}{*}{-1.55} \\
\hline & Higher education & 91 & 52.54 & 4781.00 & & \\
\hline $\begin{array}{l}\text { Frequency parents talk everyday events (once a } \\
\text { week or more often) }\end{array}$ & Higher education & 210 & 115.17 & 24185.50 & \multirow{2}{*}{2030.50} & \multirow{2}{*}{$-2.78^{* *}$} \\
\hline 2. Teen academic award & & & & & & \\
\hline \multirow{2}{*}{ Time parents help kid's homework (3hrs or more) } & Secondary education & 4 & 16.25 & 65.00 & \multirow[t]{2}{*}{45.00} & \multirow[t]{2}{*}{-.37} \\
\hline & Higher education & 25 & 14.80 & 370.00 & & \\
\hline \multirow{2}{*}{ Time parents take kids to activities (3hrs or more) } & Secondary education & 6 & 17.83 & 107.00 & \multirow[t]{2}{*}{86.00} & \multirow[t]{2}{*}{-.38} \\
\hline & Higher education & 31 & 19.23 & 596.00 & & \\
\hline Frequency parents check kid's homework (often) & Secondary education & 18 & 51.97 & 935.50 & 764.50 & -.82 \\
\hline $\begin{array}{l}\text { Frequency parents talk everyday events (once a } \\
\text { week or more often) }\end{array}$ & Higher Education & 217 & 125.12 & 27151.00 & 2795.00 & -1.14 \\
\hline
\end{tabular}

Note. Only specific cases within each parental involvement variable were selected for a comparative analysis between both educational levels (i.e., time parents help kid's homework - "3hrs or more"; time parents take kids to activities - "3hrs or more"; frequency parents check kid's homework - "often"; frequency parents talk everyday events - “once a week or more often"). ${ }^{* *} p<.01$.

\section{Discussion}

The present study explored the relationship between adolescent academic achievement and parental involvement and how parental level of education could impact adolescent academic performance when parents of all educational levels equally engage in their children's schooling.

Prior to the start of the inquiry, it was hypothesized that adolescent academic accomplishment would be positively correlated with parental involvement. Contrary to the hypothesis and the findings of existing studies [1, 17], a positive correlation was found only in certain dimensions of parental involvement. Specifically, positive correlations were identified between child's grade and the amount of time parents spend on assisting children with homework as well as between child's academic award and the frequency parents converse with children about everyday events. Frequency that parents check their child's homework was significantly positively associated with child's grade; however, surprisingly enough, it was also significantly and negatively correlated with child's academic award. The amount of time that parents spend on taking children to activities did not show any correlation with either of the two academic performance variables. Taken together, the overall findings suggest that the magnitude and direction of impact of parental involvement upon children's school achievement may vary substantially by the type of parental educational engagement.

The second hypothesis that adolescents of parents with higher levels of education would academically outperform those of parents with lower levels of education was formulated based on existing research findings that demonstrated a positive correlation between parents' education level and children's success at school [8, 18]. All in all, significant differences in terms of adolescent school performance were not observed between the two groups of parents who were equally involved in their children's education, contrary to the hypothesis. Against all expectations, however, children of parents with secondary levels of education earned higher grades as opposed to those of parents with higher levels of education when parents were often involved in talking about everyday events with their children. From these findings, it can be concluded that the overall influence of parental educational level upon children's academic achievement may be no longer significant when parents are actively involved in their child's education.

Overall, this research has several notable implications. First, the findings of this study revealed which types of parental involvement are correlated to child's school performance, and which are not. Conversely, it indicates that the potential effect of parental involvement on children's school achievement may considerably differ according to the characteristics or qualities of parental involvement (e.g., supervision-based involvement vs. communication-based involvement). Clarifying the nature of these relationships could help enhance adolescent's academic achievement as well as inform best practices for parents, educators, and policymakers. Second, to this point, limited research has been conducted on the middle-class, dual-career families in North America for this subject of study. This work holds significance in that it contributed to elevating the generalizability by expanding the boundary of the study group and adding new insights on the preexisting literature. Third, to the researcher's knowledge, it is the first inquiry attempting to define the association between parents' education level and children's school performance on the assumption that the same quantity of parental involvement is provided. This attempt brought new perspectives to the existing beliefs of parental educational attainment on children's academic achievement and laid the groundwork for future works for more rigorous exploration of this research question.

Yet, there are several limitations to this work. First, the 
survey respondents in this dataset were predominantly biological parents. Additional studies including proportionally balanced participants in terms of parents' types such as adoptive parents, foster parents, or stepparents are needed. Next, a few survey items used in this study contained a relatively high percentage of missing data. Future researchers should come up with alternative ways of adequately dealing with large proportions of missing values, where applicable, to reduce biased estimates and strengthen the generalizability of the results. Finally, the data utilized in this report was collected exclusively through self-reporting methods. Varied modes of gathering data are needed to improve the measurement quality and, in turn, produce more unbiased and valid results.

Notwithstanding, in the modern era where many science-based approaches have been attempted to enhance the quality of education, it is hoped that this manuscript contributes to prompting more active research for developing more effective pedagogical methods and learning tools. Then, it is expected that, based on these findings, educators, parents, and professionals in this field will continue making various attempts to promote life satisfaction and mental well-being of both students and their families as well as to heighten each student's potential academic competence and academic interest to an optimal level.

\section{Conclusion}

This study investigated the linkage between parental involvement, parents' level of education, and student academic success targeting particular population groups that have received scant attention in prior research. It revealed that the effect of parental involvement upon child's academic performance is not absolute but rather is contingent on the types of parental involvement. In addition, this study demonstrated that there is no influence of parents' educational level on children's school performance when adequate amounts of parental involvement and support are available to the children. As further research, replication studies with different subjects in various broader contexts are recommended to ensure the validity and reliability of these findings.

\section{References}

[1] Otani, M. (2019). Relationships between parental involvement and adolescents' academic achievement and aspiration. International Journal of Educational Research, 94, 168-182. https://doi.org/10.1016/j.ijer.2019.01.005

[2] Bloom, B. S. (1980). The new direction in education research: Alterable variables. Phi Delta Kappan, 61, 382-385.

[3] Fan, X., \& Chen, M. (2001). Parental involvement and students' academic achievement: A meta-analysis. Educational Psychology Review, 13 (1), 1-22. https://doi.org/10.1023/A:1009048817385

[4] Monteflor, M. O., Williams, A. R., Williams, P. D., Go, N. O., Moriarty, J. P., Quiñones, H. W., \& Bruggeman, S. K. (2006).
Parent motivation strategies and the performance of preschoolers in a rural Philippine municipality. Early Childhood Education Journal, 33 (5), 333-340. https://doi.org/10.1007/s10643-005-0035-1

[5] Walker, J., Ice, C., Hoover-Dempsey, K., \& Sandler, H. (2011). Latino parents' motivations for involvement in their children's schooling: An exploratory study. The Elementary School Journal, 111 (3), 409-429. https://doi.org/10.1086/657653

[6] Fu, M., Bo, W. V., Xue, Y., \& Yuan, T. (2017). Parental absence accompanies worse academic achievements: Evidence based upon a sample of left-behind children in rural China. Frontiers in Education, 2, 1-7. https://doi.org/10.3389/feduc.2017.00038

[7] Idris, M., Hussain, S., \& Ahmad, N. (2020). Relationship between parents' education and their children's academic achievement. Journal of Arts and Social Sciences, 7 (2), 82-92. https://doi.org/10.46662/jass-vol7-iss2-2020 (82-92)

[8] Khan, R., Iqbal, N., \& Tasneem, S. (2015). The influence of parents educational level on secondary school students academic achievements in District Rajanpur. Journal of Education and Practice, 6 (16), 76-79.

[9] Shoukat, A., Ilyas, M., Azam, R., \& Ch, A. H. (2013). Impact of parents' education on children's academic performance. Secondary Education Journal, 2 (1), 53-59.

[10] Eccles, J. S. (2005). Influences of parents' education on their children's educational attainments: The role of parent and child perceptions. London Review of Education, 3 (3), 191-204. https://doi.org/10.1080/14748460500372309

[11] Schneider, B., \& Waite, L. J. (2008). The 500 family study [1998-2000: United States] (ICPSR 4549; Version V1) [Data set]. ICPSR. https://doi.org/10.3886/ICPSR04549.v1

[12] Althoff, R. (2010). Dysregulated children reconsidered. Journal of the American Academy of Child \& Adolescent Psychiatry, 49 (4), 302-305. https://doi.org/10.1016/j.jaac.2010.02.003

[13] Christenson, S. L., Rounds, T., \& Gorney, D. (1992). Family factors and student achievement: An avenue to increase students' success. School Psychology Quarterly, 7 (3), 178-206. https://doi.org/10.1037/h0088259

[14] Walberg, H. J. (1986). Synthesis of research on teaching. In M. C. Wittrock (Ed.), Handbook of research on teaching (3rd ed., pp. 214-229). New York: Macmillan.

[15] Keith, T. Z., Troutman, G. C., Trivette, P. S., Keith, P. B., Bickley, P. G., \& Singh, K. (1993). Does parental involvement affect eighth-grade student achievement? Structural analysis of national data. School Psychology Review, 22 (3), 474-496. https://doi.org/10.1080/02796015.1993.12085668

[16] Gyamfi, K., \& Pobbi, M. A. (2016). Parental monitoring and child performance in Ghana. Journal of Education and Practice, 7 (21), 33-41.

[17] Jeynes, W. H. (2007). The relationship between parental involvement and urban secondary school student academic achievement: A meta-analysis. Urban Education, 42 (1), 82-110. https://doi.org/10.1177/0042085906293818

[18] Terfassa, A. D. (2018). The relationship between parental education and children's academic performance: The case of Genda Tesfa primary school, Dire Dawa. Research on Humanities and Social Sciences, 8 (5), 10-16. 